\title{
Spectral Expansion Solution Methodology for QBD-M Processes and Applications in Future Internet Engineering
}

\author{
Tien Van Do ${ }^{1}$, Ram Chakka ${ }^{2}$, and János Sztrik ${ }^{3}$ \\ 1 Department of Networked Systems and Services \\ Budapest University of Technology and Economics \\ Budapest, Hungary \\ 2 Meerut Institute of Engineering and Technology (MIET), \\ Meerut 250005, India \\ 3 Faculty of Informatics, \\ University of Debrecen, \\ Egyetem tér 1, Po.Box 12, 4010 Debrecen, Hungary
}

\begin{abstract}
Quasi Simultaneous-Multiple Births and Deaths (QBD-M) Processes are used to model many of the traffic, service and related problems in modern communication systems. Their importance is on the increase due to the great strides that are taking place in telecommunication systems and networks. This paper presents the overview of the Spectral Expansion (SE) for the steady state solution of QBD-M processes and applications in future Internet engineering.
\end{abstract}

Keywords: QBD-M, Compound Poisson Process, Spectral Expansion

\section{Introduction}

The concept of Quasi Birth-Death (QBD) processes, as a generalization of the classical birth and death $\mathrm{M} / \mathrm{M} / 1$ queues was first introduced by [1] and [2] in the late sixties. The states of a QBD process are described by two dimensional random variables called a phase and a level [3-5] and transitions in a QBD process are only possible between adjacent levels. It is observed that QBD processes create a useful framework for the performability analysis of many problems in telecommunications and computer networks [6-11].

In the QBD process, if the nonzero jumps in levels are not accompanied with changes in a phase, then these processes are known as Markovmodulated Birth and Death processes. The infinite number of states involved makes the solution of these models nontrivial. There are several methods of solving these models, either the whole class of models or any of the subclasses.

Seelen has analysed a $\mathrm{Ph} / \mathrm{Ph} / \mathrm{c}$ queue in this frame work [12]. Seelen's method is an approximate one, the Markov chain is first truncated to a finite state which is an approximation of the original process. The resulting finite state Markov 
chain is then analysed, by exploiting the structure in devising an efficient iterative solution algorithm. The second method is to reduce the infinitestate problem to a linear equation involving vector generating function and some unknown probabilities. The latter are then determined with the aid of the singularities of the coefficient matrix. A comprehensive treatment of that approach, in the context of a discretetime process with a general $\mathrm{M} / \mathrm{G} / 1$ type structure, is presented in [13]. The third way of solving these models is the well known matrixgeometric method, first proposed by Evans [2,3]. In this method a nonlinear matrix equation is first formed from the system parameters and the minimal nonnegative solution $R$ of this equation is computed by an iterative method. The invariant vec tor is then expressed in terms of the powers of $R$. Neuts claims this method has probablistic interpretation for the steps in computation. That is certainly an ad vantage. Yet, this method suffers from the fact that there is no way of knowing how many iterations are needed to compute $R$ to a given accuracy. It can also be shown that for certain parameter values the computation requirements are uncertain and formidably large. The fourth method is known as spectral expansion method. It is based on expressing the invariant vector of the process in terms of eigenvalues and left eigenvectors of a certain matrix polynomial. The generating function and the spectral expansion methods are closely related. However, the latter produces steady state probabilities directly using an algebraic expansion while the former provides them through a transform.

It is confirmed by a number of works that the spectral expansion method is better than the matrix geometric one from some aspects $[4,14,15]$. This paper gives the overview of the SE methodology and explains how the SE methodology is used towards the analysis of QBD-M processes and the performance evaluation of ICT systems and future Internet.

The rest of the paper is organized as follows. In Section 2, the terminology and definitions are presented. The spectral expansion methodology is provided in Section 3. Examples are given in Section 4. The paper is concluded in Section 5.

\section{Definitions}

Consider a two-dimensional continuous time, irreducible Markov chain $X=\{(I(t), J(t)), t \geq 0\}$ on lattice strips.

- $I(t)$ is called the phase (e.g., the state of the environment) of the system at time $t$. Random variable $I(t)$ takes values from the set $\{0,1,2, \ldots, N\}$, where $N$ is the maximum value of the phase variable.

- Random variable $J(t)$ is often called the level of the system at time $\mathrm{t}$ and takes a set of values $\{0,1, \ldots, L\}$, where $L$ can be finite or infinite.

The state space of Markov chain $X$ is $\{(i, j): 0 \leq i \leq N, 0 \leq j \leq L\}$. Let $p_{i, j}$ denote the steady state probability of the state $(i, j)$ as

$$
p_{i, j}=\lim _{t \rightarrow \infty} \operatorname{Pr}(I(t)=i, J(t)=j) ; \quad(i=0, \ldots, N ; \quad j=0,1, \ldots, L) .
$$


Vector $\mathbf{v}_{j}$ is defined as

$$
\mathbf{v}_{j}=\left(p_{0, j}, \ldots, p_{N, j}\right) \quad(j=0,1, \ldots, L) .
$$

Since the sum of all the probabilities $p_{i, j}$ is 1.0 , we have the normalization equation as

$$
\sum_{j=0}^{L} \mathbf{v}_{j} \mathbf{e}_{N+1}=1
$$

where $\mathbf{e}_{N+1}$ is a column vector of size $N+1$ with all ones.

\subsection{Continuous Time QBD Processes}

Definition 1. A continuous time Quasi-Birth-and-Death (QBD) process is formed when one-step transitions of the Markov chain $X$ are allowed to states in the same level or in the two adjacent levels. That is, the dynamics of the process are driven by

(a) purely phase transitions. $A_{j}(i, k)$ denotes the transition rate from state $(i, j)$ to state $(k, j)(0 \leq i, k \leq N ; j=0,1, \ldots, L)$;

(b) one-step upward transitions. $B_{j}(i, k)$ is the transition rate from state $(i, j)$ to state $(k, j+1)(0 \leq i, k \leq N ; j=0,1, \ldots, L)$;

(c) one-step downward transitions. $C_{j}(i, k)$ is the transition rate from state $(i, j)$ to state $(k, j-1)(0 \leq i, k \leq N ; j=0,1, \ldots, L)$.

Let $A_{j}, B_{j}$ and $C_{j}$ denote $(N+1) \times(N+1)$ matrices with elements $A_{j}(i, k)$, $B_{j}(i, k)$ and $C_{j}(i, k)$, respectively. Note that their diagonal elements are zero. Let $D^{A_{j}}, D^{B_{j}}$ and $D^{C_{j}}$ be the diagonal matrices of size $(N+1) \times(N+1)$, defined by the $i^{\text {th }}(i=0, \ldots, N)$ diagonal element as follows

$$
D^{A_{j}}(i, i)=\sum_{k=0}^{N} A_{j}(i, k) ; D^{B_{j}}(i, i)=\sum_{k=0}^{N} B_{j}(i, k) ; D^{C_{j}}(i, i)=\sum_{k=0}^{N} C_{j}(i, k) .
$$

For the convenience of the presentation we define matrices $B_{-1}=0, B_{L}=0$ and $C_{0}=0$.

The steady state balance equations satisfied by the vectors $\mathbf{v}_{j}$ are

$$
\mathbf{v}_{j}\left[D^{A_{j}}+D^{B_{j}}+D^{C_{j}}\right]=\mathbf{v}_{j-1} B_{j-1}+\mathbf{v}_{j} A_{j}+\mathbf{v}_{j+1} C_{j+1} \quad \forall j .
$$

Assume that there exist thresholds $T_{1}^{*}$ and $T_{2}^{*}$ such that

$$
\begin{aligned}
& A_{j}=A \quad\left(T_{2}^{*} \geq j \geq T_{1}^{*}\right), \\
& B_{j}=B\left(T_{2}^{*} \geq j \geq T_{1}^{*}-1\right), \\
& C_{j}=C\left(T_{2}^{*}+1 \geq j \geq T_{1}^{*}\right) .
\end{aligned}
$$

$D^{A}, D^{B}$ and $D^{C}$ are the corresponding diagonal matrices with the diagonal elements as

$$
D^{A}(i, i)=\sum_{k=1}^{N} A(i, k), D^{B}(i, i)=\sum_{k=1}^{N} B(i, k), D^{C}(i, i)=\sum_{k=1}^{N} C(i, k) .
$$


The generator matrix of the QBD process is written as

$$
\begin{aligned}
& {\left[\begin{array}{lllllllll}
A_{0}^{(1)} & B_{0} & 0 & 0 & \ldots & \ldots & \ldots & \ldots & \ldots \\
C_{1} & A_{1}^{(1)} & B_{1} & 0 & \ldots & \ldots & \ldots & \ldots & \ldots \\
0 & C_{2} & A_{2}^{(1)} & B_{2} & \ldots & \ldots & \ldots & \ldots & \ldots \\
\vdots & \vdots & \vdots & \ddots & \ldots & \ldots & \ldots & \ldots & \\
0 & 0 & \ldots & C_{T_{1}^{*}-1} & A_{T_{1}^{*}-1}^{(1)} & B_{T_{1}^{*}-1} & 0 & 0 & \ldots \\
0 & 0 & \ldots & 0 & C_{T_{1}^{*}} & A_{T_{1}^{*}}^{(1)} & B_{T_{1}^{*}} & 0 & \ldots \\
0 & 0 & \ldots & 0 & 0 & C_{T_{1}^{*}+1} & A_{T_{1}^{*}+1}^{(1)} & B_{T_{1}^{*}+1} & \ldots \\
\vdots & \vdots & \vdots & \vdots & \ldots & \ldots & \ddots & \ldots & \ldots
\end{array}\right]} \\
& =\left[\begin{array}{llllllllll}
A_{0}^{(1)} & B_{0} & 0 & 0 & \ldots & \ldots & \ldots & \ldots & \ldots \\
C_{1} & A_{1}^{(1)} & B_{1} & 0 & \ldots & \ldots & \ldots & \ldots & \ldots \\
0 & C_{2} & A_{2}^{(1)} & B_{2} & \ldots & \ldots & \ldots & \ldots & \ldots \\
\vdots & \vdots & \vdots & \ddots & \ldots & \ldots & \ldots & \ldots \\
0 & 0 & \ldots & C_{T_{1}^{*}-1} & A_{T_{1}^{*}-1}^{(1)} & Q_{0} & 0 & 0 & \ldots & \ldots \\
0 & 0 & \ldots & 0 & C_{T_{1}} & Q_{1} & Q_{0} & 0 & \ldots & \ldots \\
0 & 0 & \ldots & 0 & 0 & Q_{2} & Q_{1} & Q_{0} & \ldots \\
0 & 0 & \ldots & 0 & 0 & 0 & Q_{2} & Q_{1} & Q_{0} & \ldots \\
\vdots & \vdots & \vdots & \vdots & \ldots & \ldots & \ddots & \ldots & \ldots
\end{array}\right],
\end{aligned}
$$

where $A_{j}^{(1)}=A_{j}-D^{A_{j}}-D^{B_{j}}-D^{C_{j}}$.

The j-independent balance equations can be rewritten as follows

$$
\mathbf{v}_{j-1} Q_{0}+\mathbf{v}_{j} Q_{1}+\mathbf{v}_{j+1} Q_{2}=0 \quad\left(T_{1}^{*} \leq j \leq T_{2}^{*}\right),
$$

where $Q_{0}=B, Q_{1}=A-D^{A}-D^{B}-D^{C}, Q_{2}=C$.

\subsection{Continuous Time QBD-M Processes}

Definition 2. The Markov chain $X$ is called a continuous time quasi simultaneous-bounded-multiple births and simultaneous-bounded-multiple deaths $(Q B D-M)$ process if the balance equation for level $j$ can be written as

$$
\sum_{i=0}^{y} \mathbf{v}_{j-y_{1}+i} Q_{i}=0 \quad\left(T_{1} \leq j \leq T_{2}\right),
$$

where $y, y_{1}, T_{1}$ and $T_{2}$ are integer constants for a specific system, while $Q_{i}$ are $j$-independent matrices of size $(N+1) \times(N+1)$.

\subsection{Generalized Exponential Distribution}

Definition 3. The versatile Generalized Exponential (GE) distribution is given in the following form:

$$
F(t)=P(W \leq t)=1-(1-\phi) e^{-\mu t}(t \geq 0),
$$

where $W$ is the GE random variable with parameters $\mu, \phi$. 
Thus, the GE parameter estimation can be by obtained by $1 / \nu$, the mean, and $C_{\text {coeff }}^{2}$, the squared coefficient of variation of the inter-event time of the sample as

$$
1-\phi=2 /\left(C_{\text {coeff }}^{2}+1\right) ; \mu=\nu(1-\phi) .
$$

Remarks. For $C_{\text {coeff }}^{2}>1$, the GE model is a mixed-type probability distribution having the same mean and coefficient of variation, and with one of the two phases having zero service time, or a bulk type distribution with an underlying counting process equivalent to a Batch (or Bulk) Poisson Process (BPP) with batch-arrival rate $\mu$ and geometrically distributed batch size with mean $1 /(1-\phi)$ and SCV $\left(C_{\text {coeff }}^{2}-1\right) /\left(1+C_{\text {coeff }}^{2}\right)$ (see [16]). It can be observed that there is an infinite family of BPP's with the same GE-type inter-event time distribution. It is shown that, among them, the BPP with geometrically distributed bulk sizes (referred as the CPP) is the only one that constitutes a renewal process (the zero inter-event times within a bulk/batch are independent if the bulk size distribution is geometric [17]). The GE distribution is versatile, possessing pseudo-memoryless properties which make the solution of many GE-type queuing systems analytically tractable [17]. The choice of the GE distribution is often motivated by the fact that measurements of actual inter-arrival or service times may be generally limited and so only a few parameters (for example the mean and variance) can be computed reliably. Typically, when only the mean and variance can be relied upon, a choice of a distribution which implies least bias is that of GE-type distribution $[17,16]$.

Definition 4 (CPP). The inter-arrival time distribution of customers of the Compound Poisson Process (CPP) is GE with parameters $(\sigma, \theta)$. That is, the inter-arrival time probability distribution function is $1-(1-\theta) e^{-\sigma t}$.

Thus, the arrival point-process has batches arriving at each point having independent and geometric batch-size distribution. Specifically the probability that a batch is of size $s$ is $(1-\theta) \theta^{s-1}$.

\section{The Spectral Expansion Method for QBD-M Processes}

Let $Q(\lambda)$ denote the characteristic matrix polynomial associated with the balance equation (4) as

$$
Q(\lambda)=\sum_{i=0}^{y} Q_{i} \lambda^{i}
$$

If $(\lambda, \psi)$ is the left-eigenvalue and eigenvector pair of the characteristic matrixpolynomial, the following equation holds

$$
\psi Q(\lambda)=0 ; \operatorname{det}[Q(\lambda)]=0 .
$$

Assume that $Q(\lambda)$ has $d$ pairs of eigenvalue-eigenvectors. For the $k^{\text {th }}(k=$ $1, \ldots, d)$ non-zero eigenvalue-eigenvector pair, $\left(\lambda_{k}, \boldsymbol{\psi}_{k}\right)$, by substituting $\mathbf{v}_{j}=$ $\boldsymbol{\psi}_{k} \lambda_{k}^{j}\left(T_{1}-y_{1} \leq j \leq T_{2}-y_{1}+y\right)$ in the equations (4), it can be seen that this 
set of equations is satisfied. Hence, that is a particular solution. The equations can even be satisfied with $\boldsymbol{\psi}_{k} \lambda_{k}^{j+l_{k}}$ for any real $l_{k}$. It is easy to prove that the general solution for $\mathbf{v}_{j}$ is the linear sum of all the factors $\left(\boldsymbol{\psi}_{k} \lambda_{k}^{j-T_{1}+y_{1}}\right)$ as

$$
\mathbf{v}_{j}=\sum_{l=1}^{d} a_{l} \boldsymbol{\psi}_{l} \lambda_{l}^{j-T_{1}+y_{1}}\left(j=T_{1}-y_{1}, T_{1}-y_{1}+1, \ldots, T_{2}-y_{1}+y\right),
$$

where $a_{l}(l=1, \ldots, d)$ are constants.

Therefore, the steady state probability can be written as follows

$$
p_{i, j}=\sum_{l=1}^{d} a_{l} \boldsymbol{\psi}_{l}(i) \lambda_{l}^{j-T_{1}+y_{1}}\left(j=T_{1}-y_{1}, T_{1}-y_{1}+1, \ldots, T_{2}-y_{1}+y\right) .
$$

An interesting property can be observed concerning the eigenvalues of $Q(\lambda)$ for QBD-M process $X$ as follows. If $\left(\lambda_{k}, \psi_{k}\right)$ is the left-eigenvalue and eigenvector pair of $Q(\lambda)$, then $\left(1 / \lambda_{k}, \boldsymbol{\psi}_{k}\right)$ is the left-eigenvalue and eigenvector pair of $\bar{Q}(\lambda)=\sum_{i=0}^{y} Q_{y-i} \lambda^{i}$, the characteristic matrix polynomial of the dual process of $X($ see $[14])$.

\subsection{Infinite QBD-M Processes}

When $L$ and $T_{2}$ are infinite (unbounded), consider the probability sum

$$
\sum_{j=T_{1}-y_{1}}^{\infty} p_{i, j}=\sum_{j=T_{1}-y_{1}}^{\infty} \sum_{l=1}^{d} a_{l} \boldsymbol{\psi}_{l}(i) \lambda_{l}^{j-T_{1}+y_{1}}
$$

In order to ensure that this sum is less or equal to 1.0, the necessary condition is

$$
a_{k}=0 \text {, if }\left|\lambda_{k}\right| \geq 1 \text {. }
$$

Thus, by renumbering the eigenvalues inside the unit circle, the general solution is obtained as

$$
\begin{gathered}
\mathbf{v}_{j}=\sum_{l=1}^{\chi} a_{l} \boldsymbol{\psi}_{l} \lambda_{l}^{j-T_{1}+y_{1}} \quad\left(j=T_{1}-y_{1}, T_{1}-y_{1}+1, \ldots\right), \\
p_{i, j}=\sum_{l=1}^{\chi} a_{l} \boldsymbol{\psi}_{l}(i) \lambda_{l}^{j-T_{1}+y_{1}} \quad\left(j=T_{1}-y_{1}, T_{1}-y_{1}+1, \ldots\right) .
\end{gathered}
$$

where $\chi$ is the number of eigenvalues that are present strictly within the unit circle. These eigenvalues appear some as real and others as complex-conjugate pairs, and as do the corresponding eigenvectors.

In order to determine the steady state probabilities, the unknown constants $a_{l}$ are to be determined. Their number is $\chi$. We still have other unknowns 
$\mathbf{v}_{0}, \mathbf{v}_{1}, \ldots, \mathbf{v}_{T_{1}-y_{1}-1}$. These unknowns are determined with the aid of the state dependent balance equations (their number is $T_{1}(N+1)$ ) and the normalization equation (1), out of which $T_{1}(N+1)$ are linearly independent. These equations can have a unique solution if and only if $\left(T_{1}-y_{1}\right)(N+1)+\chi=T_{1}(N+1)$, or equivalently

$$
\chi=y_{1}(N+1)
$$

holds.

\subsection{Finite QBD-M Processes}

In order to compute the steady state probabilities, the unknown constants $a_{l}$ are to be determined. Their number is $d$. We still have other unknowns $\mathbf{v}_{0}, \mathbf{v}_{1}, \ldots, \mathbf{v}_{T_{1}-y_{1}-1}, \mathbf{v}_{T_{2}-y_{1}+y+1}, \mathbf{v}_{T_{2}-y_{1}+y+2}, \ldots, \mathbf{v}_{L}$. Therefore, the number of unknowns is

$$
d+\left(T_{1}-y_{1}\right)(N+1)+\left(L-T_{2}+y_{1}-y\right)(N+1)
$$

These unknowns are determined with the aid of the state dependent balance equations (their number is $T_{1}(N+1)+\left(L-T_{2}\right)(N+1)$ ) and the normalization equation, out of which $T_{1}(N+1)+\left(L-T_{2}\right)(N+1)$ are linearly independent. These equations can have a unique solution if and only if

$d+\left(T_{1}-y_{1}\right)(N+1)+\left(L-T_{2}+y_{1}-y\right)(N+1)=T_{1}(N+1)+\left(L-T_{2}\right)(N+1)$,

equivalently

$$
d=y(N+1)
$$

holds.

\section{Examples and Applications}

Example $1(M / M / c / L$ queue with breakdowns and repairs). The queue with an infinite buffer is described by the Markov chain $\{I(t), J(t)\}$, where $I(t)$-the operative state of the system- represents the number of operative servers at time $t$ and $J(t)$ is the number of jobs in the system at time $t$, including those being served. The maximum number of operative servers is $c$. The Markov chain is irreducible with state space $\{0,1, \ldots, c\} \times\{0,1, \ldots, L\}$. Note that in this example the phase is numbered from 0 and the transition rate matrices are of size $(c+1) \times(c+1)$. The number of phases is $N=c+1$. Jobs arrive according to an independent Poisson process with rate $\sigma$. The service rate of an operative server is denoted by $\mu$. Processors break down independently at rate $\xi$ and are repaired at rate $\eta$. When a new job arrives or when a completed job departs from the system, the operative state does not change. 
The matrices $A_{j}$ and $A$ are given by

$$
A=A_{j}=\left[\begin{array}{cccc}
0 & c \eta & & \\
\xi & 0 & (c-1) \eta & \\
2 \xi & 0 & \ddots \\
& \ddots & \ddots & \eta \\
& & c \xi & 0
\end{array}\right] \quad(j=0,1, \ldots)
$$

The one-step upward transitions are created by the arrivals of single jobs. Therefore, $B$ and $B_{j}$ the one-step upward transition rate matrices are

$$
B=B_{j}=\operatorname{diag}[\sigma, \sigma, \ldots, \sigma](j=0,1, \ldots) .
$$

The one-step downward transitions take place by the departures of single jobs, after their service completion. The departure rate $\left(C_{j}(i, i)\right)$ of jobs at time $t$ depends on $I(t)=i$ and $J(t)=j$. If $i>j$, then a server is assigned to every job and not all operative servers are occupied, hence the departure rate $C_{j}(i, i)=$ $j \mu$. If $i \leq j$, then all the operative processors are occupied by jobs, hence the departure rate $C_{j}(i, i)=i \mu$. Note that $C_{j}(i, i)$ does not depend on $j$ if $j \geq i$. Therefor, $C_{j}$ does not depend on $j$ if $j \geq c$.

$$
\begin{aligned}
C_{j} & =\operatorname{diag}[0, \min (j, 1) \mu, \min (j, 2) \mu, \ldots, \min (j, c) \mu] \quad(0<j<c), \\
C & =\operatorname{diag}[0, \mu, 2 \mu, \ldots, c \mu] \quad(j \geq c), \\
C_{0} & =0
\end{aligned}
$$

The $\mathrm{M} / \mathrm{M} / \mathrm{c} / \mathrm{L}$ queue with breakdowns and repairs is an example of the QBD process, where the coefficient matrices of the characteristic matrix polynomial are $Q_{0}=B=B=\operatorname{diag}[\sigma, \sigma, \ldots, \sigma], Q_{1}=A-D^{A}-D^{B}-D^{C}, Q_{2}=C$.

Example 2 (Retrial queues to model DHCP [18]). The size of the pool (i.e.: the number of allocatable IP addresses) is $c$. The fix lease time value sent by the DHCP server is denoted by $T_{l}$. The interarrival times of DHCP requests are exponentially distributed with a mean interarrival time $1 / \lambda$.

Assume that the holding times (i.e.: how long does a client need an IP address) of clients are represented by random variable $H$ with a cumulative distribution function $\operatorname{Pr}(H<x)=F(x)$. Upon the expiration of the lease time, the previously allocated address at the DHCP server becomes free and can be allocated to another client unless the client extends the use of a specific IP address before the expiration of the lease time. Let $a$ denote the probability that DHCP clients leave (i.e.: switch off the computer) the system or do not renew the allocated IP address after the expiration of its lease time. We can write

$$
a=\operatorname{Pr}\left(H<T_{l}\right)=F\left(T_{l}\right) .
$$

Let $I(t)$ denote the number of allocated IP addresses at time $t$. Note that $0 \leq I(t) \leq c$ holds. A client who does not receive the allocation of an IP address 
because the shortage (when $I(t)=c$ ) of IP addresses sets a timer to wait for a limited time and will retry the request for an IP address upon the expiration of backoff time. We model this phenomenon as the client joins the "virtual orbit". $J(t)$ represents the number of DHCP clients in the "orbit" at time $t$ and takes values from 0 to $\infty$.

Lease times are exponentially distributed with a mean lease time $1 / \mu=T_{l}$. Clients waiting in the orbit repeat the request for the DHCP server with rate $\nu$ (i.e.: the inter-repetition times are exponentially distributed with parameter $\nu$ ), which is independent from the number of waiting clients in the orbit.

The evolution of the system is driven by the following transitions.

(a) $A_{j}(i, k)$ denotes a transition rate from state $(i, j)$ to state $(k, j)(0 \leq i, k \leq$ $c ; j=0,1, \ldots)$, which is caused by either the arrival of DHCPDISCOVERY requests or by the expiration of the lease time without the renewal of an allocated IP address. Matrix $A_{j}$ is defined as the matrix with elements $A_{j}(i, k)$. Since $A_{j}$ is $j$-independent, it can be written as

$$
A_{j}=A=\left[\begin{array}{lllllll}
0 & \lambda & 0 & \ldots & 0 & 0 & 0 \\
a \mu & 0 & \lambda & \ldots & 0 & 0 & 0 \\
\vdots & \vdots & \vdots & \vdots & \vdots & \vdots & \vdots \\
0 & 0 & \ldots & a(c-1) \mu & 0 & \lambda \\
0 & 0 & \ldots & 0 & a c \mu & 0
\end{array}\right] \forall j \geq 0
$$

(b) $B_{j}(i, k)$ represents one step upward transition from state $(i, j)$ to state $(k, j+1)(0 \leq i, k \leq c ; j=0,1, \ldots)$, which is due to the arrival of DHCPDISCOVERY requests when no free IP address is available in the IP address pool. In the similar way, matrix $B_{j}(B)$ with elements $B_{j}(i, k)$ is defined as

$$
B_{j}=B=\left[\begin{array}{ccccccc}
0 & 0 & 0 & \ldots & 0 & 0 & 0 \\
0 & 0 & 0 & \ldots & 0 & 0 & 0 \\
\vdots & \vdots & \vdots & \vdots & \vdots & \vdots & \vdots \\
0 & 0 & \ldots & 0 & 0 & 0 \\
0 & 0 & \ldots & 0 & 0 & \lambda
\end{array}\right] \forall j \geq 0
$$

(c) $C_{j}(i, k)$ is the transition rate from state $(i, j)$ to state $(k, j-1)(0 \leq i, k \leq$ $c ; j=1, \ldots)$, which is due to the successful retrial of a request from the orbit. Matrix $C_{j}(\forall j \geq 1)$ with elements $C_{j}(i, k)$ is written as

$$
C_{j}=C=\left[\begin{array}{ccccccc}
0 & \nu & 0 & \ldots & 0 & 0 & 0 \\
0 & 0 & \nu & \ldots & 0 & 0 & 0 \\
\vdots & \vdots & \vdots & \vdots & \vdots & \vdots & \vdots \\
0 & 0 & \ldots & 0 & 0 & \nu \\
0 & 0 & \ldots & 0 & 0 & 0
\end{array}\right] \forall j \geq 1
$$


The infinitesimal generator matrix of $Y$ can be written as follows

$$
\left[\begin{array}{lllllll}
A_{00} & \mathrm{~B} & 0 & \ldots & \ldots & \ldots & \ldots \\
\mathrm{C} & Q_{1} & \mathrm{~B} & 0 & \ldots & \ldots & \ldots \\
0 & \mathrm{C} & Q_{1} & \mathrm{~B} & 0 & \ldots & \ldots \\
0 & 0 & \mathrm{C} & Q_{1} & \mathrm{~B} & 0 & \ldots \\
\vdots & \vdots & \vdots & \vdots & \vdots & \vdots & \vdots \\
\ldots & \ldots & \ldots & \ldots & \ldots & \ldots & \ldots
\end{array}\right]
$$

where $D^{A}$ and $D^{C}$ are diagonal matrices whose diagonal elements are the sum of the elements in the corresponding row of $A$ and $C$, respectively. Note that $A_{00}=A-D^{A}-B, Q_{1}=A-D^{A}-B-D^{C}$.

\section{Conclusions}

We have presented an overview for the spectral expansion method to solve QBD$\mathrm{M}$ processes which can be applied to evaluate the performance of various systems, services in information and communication technology (ICT)systems and future Internet. The spectral expansion method is proved to be a mature technique for the performance analysis of various problems $[4,6,7,14,19-38]$. The examples include the performance evaluation of Optical Burst/Packet (OBS) Switching networks [24,39], MPLS networks [23, 30], the Apache web server [7], and wireless networks $[6,24,28,40]$.

\section{Acknowledgement}

The publication was supported by the TÁMOP-4.2.2.C-11/1/KONV-20120001 project. The project has been supported by the European Union, cofinanced by the European Social Fund.

\section{References}

1. V. L. Wallace. The Solution of Quasi Birth and Death Processes Arising from multiple Access Computer Systems. PhD thesis, University of Michigan, 1969.

2. R. V. Evans. Geometric Distribution in some Two-dimensional Queueing Systems. Operations Research, 15:830-846, 1967.

3. M. F. Neuts. Matrix Geometric Soluctions in Stochastic Model. Johns Hopkins University Press, Baltimore, 1981.

4. I. Mitrani and R. Chakka. Spectral expansion solution for a class of Markov models: Application and comparison with the matrix-geometric method. Performance Evaluation, 23:241-260, 1995.

5. G. Latouche and V. Ramaswami. Introduction to Matrix Analytic Methods in Stochastic Modeling. ASA-SIAM Series on Statistics and Applied Probability, 1999.

6. R. Chakka and T. V. Do. The MM $\sum_{k=1}^{K} C P P_{k} / G E / c / L G$-Queue with Heterogeneous Servers: Steady state solution and an application to performance evaluation. Performance Evaluation, 64:191-209, March 2007. 
7. T. V. Do, U. R. Krieger, and R. Chakka. Performance modeling of an apache web server with a dynamic pool of service processes. Telecommunication Systems, 39(2):117-129, 2008.

8. U. R. Krieger, V. Naoumov, and D. Wagner. Analysis of a Finite FIFO Buffer in an Advanced Packet-Switched Network. IEICE Trans. Commun., E81-B:937-947, 1998.

9. V. Naoumov, U. R. Krieger, and D. Warner. Analysis of a Multi-Server DelayLoss System With a General Markovian Arrival Process. In S. R. Chakravarthy and A. S. Alfa, editors, Matrix-analytic methods in stochastic models, volume 183 of Lecture Notes in Pure and Applied Mathematics, pages 43-66. Marcel Dekker, September 1997.

10. E. Rosti, E. Smirni, and K. C. Sevcik. On processor saving scheduling policies for multiprocessor systems. IEEE Trans. Comp, 47:47-2, 1998.

11. A. Wierman, T. Osogami, M. Harchol-Balter, and A. Scheller-Wolf. How many servers are best in a dual-priority M/PH/k system? Perform. Eval., 63(12):1253$1272,2006$.

12. L. P. Seelen. An Algorithm for $\mathrm{Ph} / \mathrm{Ph} / \mathrm{c}$ queues. European Journal of Operational Research, 23:118-127, 1986.

13. H. R. Gail, S. L. Hantler, and B. A. Taylor. Spectral analysis of M/G/1 type Markov chains. Technical Report RC17765, IBM Research Division, 1992.

14. R. Chakka. Performance and Reliability Modelling of Computing Systems Using Spectral Expansion. PhD thesis, University of Newcastle upon Tyne (Newcastle upon Tyne), 1995.

15. W. K. Grassmann and S. Drekic. An analytical solution for a tandem queue with blocking. Queueing System, (1-3):221-235, 2000.

16. C. Skianis and D. Kouvatsos. An Information Theoretic Approach for the Performance Evaluation of Multihop Wireless Ad Hoc Networks. In D. D. Kouvatsos, editor, Proceedings of the Second International Working Conference on Performance Modelling and Evaluation of Heterogeneous Networks (HET-NETs 04), pages P81/1-13, Ilkley, UK, July 2004.

17. D. D. Kouvatsos. A maximum entropy analysis of the G/G/1 Queue at Equilibrium. Journal of Operations Research Society, 39:183-200, 1998.

18. T. V. Do. An Efficient Solution to a Retrial Queue for the Performability Evaluation of DHCP. Computers \& OR, 37(7):1191-1198, 2010.

19. R. Chakka. Spectral Expansion Solution for some Finite Capacity Queues. Annals of Operations Research, 79:27-44, 1998.

20. R. Chakka and P. G. Harrison. Analysis of MMPP/M/c/L queues. In Proceedings of the Twelfth UK Computer and Telecommunications Performance Engineering Workshop, pages 117-128, Edinburgh, 1996.

21. R. Chakka and P. G. Harrison. A Markov modulated multi-server queue with negative customers - the MM CPP/GE/c/L G-queue. Acta Informatica, 37:881919, 2001.

22. R. Chakka and P. G. Harrison. The MMCPP/GE/c queue. Queueing Systems: Theory and Applications, 38:307-326, 2001.

23. R. Chakka and T. V. Do. The $M M \sum_{k=1}^{K} C P P_{k} / G E / c / L$ G-Queue and Its Application to the Analysis of the Load Balancing in MPLS Networks. In 27th Annual IEEE Conference on Local Computer Networks (LCN 2002), 6-8 November 2002, Tampa, FL, USA, Proceedings, pages 735-736, 2002.

24. R. Chakka, T. V. Do, and Z. Pandi. A Generalized Markovian Queue and Its Applications to Performance Analysis in Telecommunications Networks. In D. Kou- 
vatsos, editor, Performance Modelling and Analysis of Heterogeneous Networks, pages 371-387. River Publisher, 2009.

25. R. Chakka, E. Ever, and O. Gemikonakli. Joint-state modeling for open queuing networks with breakdowns, repairs and finite buffers. In 15th International Symposium on Modeling, Analysis, and Simulation of Computer and Telecommunication Systems (MASCOTS), pages 260-266. IEEE Computer Society, 2007.

26. R. Chakka and I. Mitrani. Multiprocessor systems with general breakdowns and repairs. In SIGMETRICS, pages 245-246, 1992.

27. R. Chakka and I. Mitrani. Heterogeneous multiprocessor systems with breakdowns: Performance and optimal repair strategies. Theor. Comput. Sci., 125(1):91-109, 1994.

28. T. V. Do, R. Chakka, and P. G. Harrison. An integrated analytical model for computation and comparison of the throughputs of the UMTS/HSDPA user equipment categories. In MSWiM '07: Proceedings of the 10th ACM Symposium on Modeling, analysis, and simulation of wireless and mobile systems, pages 45-51, New York, NY, USA, 2007. ACM.

29. T. V. Do, N. H. Do, and R. Chakka. Performance evaluation of the high speed downlink packet access in communications networks based on high altitude platforms. In K. Al-Begain, A. Heindl, and M. Telek, editors, ASMTA, volume 5055 of Lecture Notes in Computer Science, pages 310-322. Springer, 2008.

30. T. V. Do, D. Papp, R. Chakka, and M. X. T. Truong. A Performance Model of MPLS Multipath Routing with Failures and Repairs of the LSPs. In D. Kouvatsos, editor, Performance Modelling and Analysis of Heterogeneous Networks, pages 2743. River Publisher, 2009.

31. S. Drekic and W. K. Grassmann. An eigenvalue approach to analyzing a finite source priority queueing model. Annals OR, 112(1-4):139-152, 2002.

32. E. Ever, O. Gemikonakli, and R. Chakka. A mathematical model for performability of beowulf clusters. In Annual Simulation Symposium, pages 118-126. IEEE Computer Society, 2006.

33. E. Ever, O. Gemikonakli, and R. Chakka. Analytical modelling and simulation of small scale, typical and highly available beowulf clusters with breakdowns and repairs. Simulation Modelling Practice and Theory, 17(2):327-347, 2009.

34. W. K. Grassmann. The use of eigenvalues for finding equilibrium probabilities of certain markovian two-dimensional queueing problems. INFORMS Journal on Computing, 15(4):412-421, 2003.

35. W. K. Grassmann and S. Drekic. An analytical solution for a tandem queue with blocking. Queueing Syst., 36(1-3):221-235, 2000.

36. I. Mitrani. Approximate solutions for heavily loaded markov-modulated queues. Perform. Eval., 62(1-4):117-131, 2005.

37. H. T. Tran and T. V. Do. Computational Aspects for Steady State Analysis of QBD Processes . Periodica Polytechnica, Ser. El. Eng, pages 179-200, 2000.

38. Y. Zhao and W. K. Grassmann. A numerically stable algorithm for two server queue models. Queueing Syst., 8(1):59-79, 1991.

39. T. V. Do and R. Chakka. A New Performability Model for Queueing and FDLrelated Burst Loss in Optical Switching Nodes. Computer Communications, 33(S):146-151, 2010.

40. T. V. Do, R. Chakka, N. Do, and L. Pap. A Markovian queue with varying number of servers and applications to the performance comparison of HSDPA user equipment. Acta Informatica, 48:243-269, 2011. 